contact lens. The eye is moulded in mouldite, and a semi-finished lens is produced to correspond with this mould. This is modified by grinding to give an exact fit. The formation of Sattler's veil and the probable causes are described in detail, but no mention is made of fenestration as a possible solution of this problem. This book contains much that is of interest to all ophthalmologists, and much that is of value to all students of contact lenses.

\title{
CORRESPONDENCE
}

\section{CONTACT GLASSES AND VEILING}

To the Editors of THE BRITISH Journal OF OPHTHALMOLOGY.

DEAR SIRS, - If the cornea and adjacent conjunctiva is examined with the slit-lamp immediately after a contact lens is removed when veiling is well established it will be seen that this condition is an oedema of both the cornea and conjunctiva which are not supported by (that is, are not in contact with) the lens. If a contact lens containing paraffin wax melting at body temperature is inserted and left in for an hour or so in a case which easily develops veiling, and the wax is chilled to set it before removing the lens, a plaster model of the eye may easily be made. Such a cast shows a heaped up frill of conjunctiva surrounding the cornea and tending to fill the space between the eye and the lens, where a pool of fluorescein would appear when fitting. If a channel, say $\frac{1}{2} \mathrm{~mm}$. wide and $\frac{1}{4} \mathrm{~mm}$. deep, is cut leading away from the corneal segment of the contact lens and on the inner surface and the lens worn until veiling occurs, when the lens is removed the eye will show an oedematous frill of conjunctiva around the limbus and also that the conjunctiva is herniated into the channel. This raised conjunctiva is easily seen with the naked eye. It is generally recognised that a lens under which veiling has developed "sticks" and is difficult to remove and it can also be shown that at this time the space between the lens and the cornea is less than it was when the lens was inserted.

These facts seem compatible only with the supposition that veiling is due to oedema of the unsupported eye tissues under a contact lens and is due to direct physical suction. This negative pressure may be developed as follows:-a lens with corneal clearance but with an edge fit, when pressed (by the lids) against the globe creates a positive pressure in the fluid under the lens which easily escapes at the lens edge. When the pressure is released, however, the lens edge acts as a valve and neither air nor 
tears can flow in to fill the space which tends to reform under the lens as the eyeball tends to resume its normal shape. A negative pressure is thus created and with each successive lid closure, especially if the lids are tightly squeezed, more fluid is expelled and a 'greater negative pressure created. This negative pressure may be accommodated in two ways. First by the actual herniation of the conjunctiva which is mobile and secondly by the passage of lymph into the tissue spaces; this oedema is first seen immediately under the epithelium. The system does not impede the centripetal flow of lymph.

It seemed to me, some years ago, that this must be the explanation of veiling - the contact lens acts as a cupping glass-but only recently have I been able to demonstrate the truth of this assumption. It is well known that a badly fitting lens, or one with a suitably drilled hole, will not cause veiling. If instead of these means a channel of suitable dimensions. and location is cut on the underside of the contact lens from the lens edge to the pre-corneal space veiling is relieved and bubbles need not be introduced. The channels may be open to the under surface or designed within the body of the material of the lens.

This procedure has proved successful in twelve consecutive cases' which I have recently fitted with moulded plastic lenses. I am putting forward this explanation of the condition and the method of dealing with it based upon this explanation, so that others may make use of them. More work will be needed before it is possible to define exactly the technical details as to form, position and number of the channels required. This I hope to make the subject of a formal communication in the future but it seems unjustifiable tó withhold an explanation and a method of cure which may be of naterial assistance to others while the whole problem is being. worked out.

Yours sincerely,

FREDERICK RIDLEY.

80, HARLEY STREET,

LONDON, W.1

November 19, 1947.

\section{OBITUARY}

\section{MacGILLIVRAY of MacGILLIVRAY}

MACGILlivRAY of MacGillivray, after a long and distinguished career as an ophthalmic surgeon in Dundee, died on October 15, 1947 at the age of 82 years. He was ophthalmic surgeon to the Dundee Koyal Infirmary for over 30 years, and surgeon to the 